\title{
Warming up with Pressure Improves Subsequent Clutch Performance on a Golf-Putting Task
}

\author{
Desmond McEwan ${ }^{1}$, Rodney Schmaltz ${ }^{2}$, Kathleen A. Martin Ginis ${ }^{1}$ \\ ${ }^{1}$ Department of Kinesiology, McMaster University, Hamilton, Canada \\ ${ }^{2}$ Department of Psychology, Grant MacEwan University, Edmonton, Canada \\ Email: mcewand@mcmaster.ca
}

Received August 25 $5^{\text {th }}, 2012$; revised September $28^{\text {th }}, 2012$; accepted October $11^{\text {th }}, 2012$

\begin{abstract}
The purpose of the current study was to determine if athletic performance in a high-pressure situation is improved by briefly warming up under high-pressure conditions. Participants first completed a warm up round of golf putting (five shots) under low, moderate, or high pressure. Following a short break, participants completed a single putt under high pressure. Participants who completed the warm up under high pressure performed significantly better on the subsequent high-pressure shot than those who warmed up under low pressure. Warming up under pressure may be an effective means of improving performance in an impending high-pressure situation.
\end{abstract}

Keywords: Choking; Pressure; Psychology; Golf; Explicit Monitoring

\section{Introduction}

The phenomenon known as "choking under pressure" has been subject to extensive experimental research in sport and performance psychology. Choking occurs when an individual performs poorer under instances of high pressure (or in "clutch" situations) in comparison to his/her performance under instances of lower pressure (Beilock \& Gray, 2007). There are certainly times when athletes do not perform at their peak but, in many instances, these performance decrements occur during critical points of competition when the importance of performing well is very high. For instance, a golfer may find that successfully sinking a short putt on the final hole of a major to win the tournament is far more daunting than other putts taken during competition.

Numerous theories have been proposed and tested to determine why high pressure can impair performance. Among these is the explicit monitoring theory, which posits that high-pressure situations raise self-consciousness and anxiety regarding performance (Baumeister, 1984; Beilock \& Gray, 2007). In these clutch situations, individuals try to exert more explicit control and monitoring than normally would be applied (and required) in lower-pressure situations. Several studies have provided support for this theory as a potential explanation for choking (e.g., Baumeister, 1984; Beilock \& Carr, 2001; Lewis \& Linder, 1997; Masters, 1992) and suggest that the psychological demands - specifically the self-regulatory demands - are different in high-pressure situations of competition compared to other situations. In addition, research guided by the explicit monitoring theory has been conducted to test for potential ways to ameliorate choking. Most pertinent to the current study is the finding that an athlete's learning condition can affect his/her performance in later high-pressure situations. Beilock and Carr (2001) found that participants who were trained on a golf-putting task under self-consciousness-evoking learning conditions were less likely to choke on a high-pressure follow-up task that would increase self-consciousness compared to those who had been trained under conditions that did not evoke self-consciousness. The researchers concluded that these participants performed better because they had been trained under conditions similar to the final testing situation. In addition, other studies have found that individuals who train under conditions of mild anxiety are more likely to perform successfully in later high-pressure situations compared to those who train without anxiety (Oudejans \& Pijpers, 2010; Oudejans \& Pijpers, 2009). Thus, it seems that individuals are better prepared for performing in situations that are similar to those they have encountered previously, as they seem to have become "accustomed" to the self-regulatory demands of these types of situations (Baumeister, 1984).

In the current study, we sought to build upon the results of the aforementioned studies of performing in high-pressure situations. While the training environment that an athlete practices in is clearly an important factor for improving performance under high pressure, we believe that preparation should also occur just prior to these high-pressure scenarios (i.e., during an individual's warm up). As Baumeister, Heatherton, and Tice (1994: p. 19) put it: "people who are not accustomed to controlling themselves should find it difficult to do so when it suddenly becomes necessary." We also believe that this type of a warm up to pressure need not be extensive. Rather, even a brief simulation of pressure will be beneficial to individuals' performance in a subsequent high-pressure situation as it will help prime the self-regulatory processes required for performing optimally in these latter situations. We tested this possibility in the present experiment using a golf-putting task. Participants first completed a series of warm up shots under low, moderate, or high pressure. Thereafter, all participants completed a single, high-pressure putt, as a simulation of the clutch situations that often result in choking in sports. Presumably, those who had recently experienced these high-pressure scenarios (i.e., those in the high-pressure warm up condition) would be better able to self-regulate under these conditions and, 
thus, perform better than those who had not recently experienced putting under high pressure (i.e., those in the low-pressure warm up condition).

It was hypothesized that the warm up condition would be a significant predictor of performance on the single, high-pressure shot. Specifically, participants who warmed up in the highpressure condition would be more likely to make the highpressure shot than those in the moderate-pressure group who, in turn, would be more likely to make the high-pressure shot than those in the low-pressure group. We believed that these differences would emerge because those who had already performed in a high-pressure situation would become accustomed to the psychological demands of performing in these high-pressure circumstances.

\section{Method}

\section{Participants}

The study sample consisted of 119 undergraduate students (28 males, 91 females) from a Canadian university who received credit toward an introductory psychology course requirement participated in this study. Ethical approval for the study was given from the university's research ethics board.

\section{Procedures}

After signing a consent form, participants were given an overview of the procedure and asked to take five practice shots to become familiar with the putter and the putting green. The putting task was completed with a neutral-handed putter and regulation golf ball on a nine-foot long indoor, carpeted putting green, which had a regulation-sized cup. The actual length of all putts was 7 feet. Although there was only one participant per session, participants were led to believe that three other individuals were completing the same study at the same time in separate rooms within the testing laboratory with another experimenter. The reason given for this separation of participants was that there was not enough room in the present room for all four participants; in actuality, this cover story was given to set up the later manipulation.

Participants were randomly allocated to the low- $(n=39)$, moderate- $(n=38)$, or high-pressure $(n=42)$ warm up condition and read through a form that provided more detailed information about the experimental task. Written instructions were provided in order to keep the experimenter blind to the condition and, thus, prevent any potential demand awareness effects. The experimenter left the room for two minutes and, upon return, asked if the participants read and understood the procedure form - there were no instances in which a participant did not understand clearly and, thus, no instances where an experimenter was clued into which group a participant had been assigned to.

Warm up round of putting. Participants were given one of three pieces of information depending on which warm up condition they were in. In the low-pressure condition, participants read that the study was being conducted to determine how many putts an average student can make out of five attempts. In the moderate-pressure condition, participants read that he/she had been paired with one of the other alleged study participants and competing against the other two alleged participants; as such, the social pressure to perform well (i.e., not letting one's teammate down) was higher in this condition compared to the low-pressure condition. The participant was told that there would be a series of rounds of five putts; his/her team's score would be compared against the other team's score and the winning team would receive a ten-dollar prize; this potential monetary reward was also included to increase the importance of performing well compared to the low-pressure condition. In the high-pressure condition, the participant read a similar procedure form as those participants in the moderate condition; however, he/she was told that there would only be one round of putting. This was included to increase the importance of performing well compared to the moderate-pressure condition. That is, while participants in the moderate-pressure condition would supposedly have many chances to perform well (in which case, they could "make up" for any poor performances over several rounds), participants in the high-pressure would only have one round of putting to perform well. Moreover, the participant was told that the other three participants had already completed their putts and his/her current performance would decide who the winners would be. Upon completion of the first five putts, the experimenter exited the room to allegedly tally up the results.

The tiebreaker scenario. After two minutes, the experimenter returned and handed the participant another procedure form. This form described that there had been a tie between the two teams and he/she was required to take a final shot to decide the competition's winners. The cover story varied slightly to correspond with the information presented in the initial round of putting. As such, participants in the low-pressure condition were informed that, contrary to what they were previously told, they had actually been paired with another participant and were competing against two other participants for a ten-dollar prize. Essentially, they were told what participants in the high-pressure condition were originally told and that a tie resulted from this alleged competition. Participants in the moderate-pressure group were informed that, instead of several rounds of putting, the previous five putts were the only ones that would count towards their final score but there was a tie between the two teams. Participants in the high-pressure condition read that the initial round of putting did not decide a winner because of a tie. All participants were then told that there was only time for one shot because the experimental session needed to be completed shortly. Therefore, if the participant made the shot, his/her team would receive the ten-dollar reward (five dollars each); however, if he/she missed, no reward would be given. Upon completion of the tiebreaker shot, participants were asked how much pressure and anxiety they felt throughout the experiment, probed for suspicions regarding the true purpose of the study, and debriefed. To maintain ethical standards, all participants were given the five-dollar reward, regardless of their performance on this shot.

\section{Data Analysis}

A one-way ANOVA was first carried out to determine whether conditions differed significantly in shot percentages on the initial five warm-up putts. A binary logistic regression was then conducted to determine whether the condition in which participants completed the five warm up shots predicted performance on the tiebreaker shot. The outcome variable was whether participants made or missed this tiebreaker shot. The predictor variable was condition in the warm up round of putting. 


\section{Results}

The shot percentage for the low-pressure condition in the warm up round was $34.4 \%$, compared to $33.2 \%$ for the moderate-pressure condition, and $37.1 \%$ for the high-pressure condition. A one-way ANOVA revealed that successful shot percentages on the five warm up shots were not significantly different between the three conditions (i.e., regardless of condition, participants were equally likely to make a given shot during the warm up), $F(118)=.25, p=.78$. Ten out of 39 participants $(25.6 \%)$ in the low-pressure condition successfully made the subsequent tiebreaker shot, compared to 13 out of 38 participants $(34.2 \%)$ in the moderate-pressure condition, and 20 out of 42 participants in the high-pressure condition. The binary logistic regression revealed that condition was a significant predictor of performance on the tiebreaker shot. As hypothesized, participants in the high-pressure warm up condition were more likely to make the tiebreaker shot than participants in the low-pressure warm up condition, Wald $=4.09, \beta=.97, p=.043$. Contrary to hypothesis, participants in the moderate-pressure condition did not perform significantly better than those in the low-pressure condition, Wald $=.67, \beta=.41, p=.413$.

\section{Discussion}

The purpose of this study was to determine whether warming up under high pressure would predict better performance on a subsequent high-pressure athletic task compared to warming up under moderate or low pressure. In partial support of our hypothesis, participants who warmed up under high pressure performed significantly better on the high-pressure tiebreaker shot than those who completed the warm up under low pressure. This finding provides an important contribution to the literature on performing under pressure. Specifically, it suggests that a brief warm up under pressure may be a beneficial way for improving performance in subsequent clutch situations.

The results of this study complement the findings of previous studies, which have shown that training in an environment that simulates the high-pressure circumstances that occur during athletic competition results in improved performance when these situations subsequently arise (Beilock \& Carr, 2001; Oudejans \& Pijpers, 2009; Oudejans \& Pijpers, 2010). In addition to training for high-pressure situations (i.e., during practices), our results indicate that it seems beneficial to briefly warm up for these situations (i.e., just prior to when they are expected to occur). Furthermore, as our initial round of putting consisted of a mere five shots, it seems that this warm up need not be overly exhaustive. As such, athletes - as well as coaches and/or sport psychologists - could easily employ this technique without interfering with the normal preparation for competition. Preparing for high-pressure situations can be viewed as analogous to preparing oneself, physically. That is, to ensure high athletic performance, a muscle or physical skill must first be trained in a manner that simulates the physical demands that will occur during an upcoming competition. Then, just prior to the competition, the athlete must stretch and warm up to also help ensure optimal performance. In much the same way, preparation for the psychological demands of a forthcoming high-pressure competition should occur during training and also while warming up immediately before the competition.

This study provides further support for the utility of the explicit monitoring theory as a guiding framework for improving athletic performance under pressure. This theory posits that, under pressure, self-consciousness increases and individuals attend to the step-by-step processes involved in completing a motor task, which impairs performance (Baumeister, 1984; Beilock \& Gray, 2007). However, this theory also suggests that familiarizing oneself with the conditions that will result in this conscious control of the skill is a beneficial way of improving performance under pressure (Baumeister, 1984). Indeed, the psychological demands on an athlete to perform well under high pressure are different than in normal competitive situations (Beilock \& Carr, 2001; Beilock \& Gray, 2007). By preparing for these demands in training and just prior to competition athletes can become better able to deal with the self-regulatory demands of high-pressure circumstances. In turn, this preparation can result in improved performance during clutch situations.

\section{Limitations}

While the results of this study provide a novel contribution to the literature of performance under pressure, it is not without its limitations. For one, our sample consisted of undergraduate psychology students. As such, it may be premature to suggest that these results generalize to athletes. Secondly, while the purpose of not having participants interact with other alleged competitors (e.g., a study confederate) in the experiment was to prevent any group dynamics effects, the resulting manner in which the current study was conducted is somewhat artificial compared to real-world sporting competitions. Thirdly, while being debriefed, most participants in the high-pressure warm up condition claimed that they felt high pressure to perform well, while most in the low-pressure condition indicated that they felt little or no pressure to perform well. However, a more rigorous manipulation check would be valuable to help quantify the amount of pressure (or other emotions such as anxiety) that participants may have experienced.

\section{Future Directions}

Discovering the most beneficial ways in which athletes can perform optimally in high-pressure situations continues to be a focus for numerous researchers in the field of sport psychology. The results from the current study are the first to suggest that it may be helpful for athletes to warm up under conditions that simulate the impending high-pressure situations that will arise during competition, and it would be valuable that additional tests of this hypothesis be carried out. For instance, it may prove beneficial to test this hypothesis with elite athletes to confirm that these results generalize to this population. Also, our warm up was quite brief, consisting of only five putts. Although this was intentional to show that warming up for subsequent high-pressure situations need not be extensive, it would be valuable to determine the ideal length of time of a warm up (i.e., the dose-response relationship between warming up and subsequent performance under pressure). Future research should also assess the combination of the optimal training and warm up situations that best replicate the experience of high-pressure competitions and, in turn, improve performance in actual competitions, thereafter.

\section{REFERENCES}

Baumeister, R. F. (1984). Choking under pressure: Self-consciousness 


\section{MCEWAN ET AL.}

and paradoxical effects of incentives on skillful performance. Journal of Personality and Social Psychology, 46, 610-620.

doi:10.1037//0022-3514.46.3.610

Baumeister, R. F., Heatherton, T. F., \& Tice, D. M. (1994). Losing control. San Diego, CA: Academic Press.

Beilock, S. L., \& Carr, T. H. (2001). On the fragility of skilled performance: What governs choking under pressure? Journal of EXperimental Psychology: General, 130, 701-725.

doi:10.1037//0096-3445.130.4.701

Beilock, S. L., \& Gray, R. (2007). Why do athletes "choke" under pressure? In G. Tenenbaum, \& R. C. Eklund (Eds.), Handbook of sport psychology (pp. 425-444). Hoboken, NJ: John Wiley \& Sons.

Lewis, B., \& Linder, D. (1997). Thinking about choking? Attentional processes and paradoxical performance. Personality \& Social Psychology Bulletin, 23, 937-944. doi:10.1177/0146167297239003

Masters, R. S. W. (1992). Knowledge, knerves, and know-how: The role of explicit versus implicit knowledge in the breakdown of a complex motor skill under pressure. British Journal of Psychology, 83, 343-358. doi:10.1111/j.2044-8295.1992.tb02446.x

Oudejans, R. R. D., \& Pijpers, J. R. (2009). Training with anxiety has a positive effect on expert perceptual-motor performance under pressure. Quarterly Journal of Experimental Psychology, 62, 1631-1647. doi:10.1080/17470210802557702

Oudejans, R. D., \& Pijpers, J. R. (2010). Training with mild anxiety may prevent choking under higher levels of anxiety. Psychology of Sport and Exercise, 11, 44-50. doi:10.1016/j.psychsport.2009.05.002 